OPEN ACCESS

Edited by:

Leandro F. Malloy-Diniz,

Universidade Federal de Minas

Gerais, Brazil

Reviewed by:

Martin Brüne,

Ruhr University Bochum, Germany

Giuliano E. Ginani,

Faculdade União das Américas, Brazi

*Correspondence:

Damien Brevers,

Department of Psychology, Brain and Creativity Institute, University

of Southern California,

3620A McClintock Avenue, Los Angeles, CA 90089-2921, USA

brevers@usc.edu

Specialty section: This article was submitted to Psychopathology,

a section of the journal

Frontiers in Psychology

Received: 16 March 2015

Accepted: 29 June 2015

Published: 09 July 2015

Citation:

Brevers D, Noël X, Hanak C, Verbanck

$P$ and Kornreich $C$ (2015) On

the relationship between emotional

state and abnormal unfairness

sensitivity in alcohol dependence.

Front. Psychol. 6:983.

doi: 10.3389/fpsyg.2015.00983

\section{On the relationship between emotional state and abnormal unfairness sensitivity in alcohol dependence}

\author{
Damien Brevers ${ }^{1,2 *}$, Xavier Noël ${ }^{2}$, Catherine Hanak ${ }^{2}$, Paul Verbanck ${ }^{2}$ and Charles \\ Kornreich $^{2}$ \\ 'Department of Psychology, Brain and Creativity Institute, University of Southern California, Los Angeles, CA, \\ USA, ${ }^{2}$ Psychological Medicine Laboratory, Faculty of Medicine, Université Libre de Bruxelles, Brussels, Belgium
}

Recent empirical findings suggest that alcohol dependence is characterized by heightened sensitivity to unfairness during social transactions. The present study went a step further and aimed to ascertain whether this abnormal level of sensitivity to unfairness is underlined by an increased emotional reactivity. Twenty-six recently abstinent alcoholdependent $(\mathrm{AD})$ individuals and 32 controls performed an ultimatum game (UG), in which participants had to respond to take-it-or-leave-it offers, ranging from fair to unfair and made by a fictive proposer. Emotional state was recorded during UG offers presentation and was indexed by the amplitude of skin conductance response (SCR). Results showed that AD decided to reject unfair offers more frequently than their controls, confirming previous data. The proportion of rejected unfair UG offers was correlated with SCR, in the AD but not in the control group. This finding suggests that deciding to accept or reject unfair UG offers is influenced by arousal-affective activity in AD, but not in controls. Heightened emotional reactivity may have driven AD to punish the proposer rather than acting as a rational economic agent. An implication of present findings is that $A D$ might have difficult to cope with unfair situations triggered by social interactions. Future studies are needed in order to examine whether-emotional and behavioral-reactivity to unfairness during the UG could impact alcohol consumption and relapse in $A D$.

Keywords: alcohol dependence, social decision-making, unfairness, electrodermal response

\section{Introduction}

Alcohol-dependent $(\mathrm{AD})$ individuals persevere in alcohol use despite encountering long-term aversive consequences directly linked to their drinking (Noël et al., 2010; American Psychiatric Association, 2013; Camchong et al., 2014). The persistence of such maladaptive habits might be underlined by poor decision-making ability. Indeed, abnormal profiles of decision-making have been repeatedly evidenced in laboratory settings, wherein recently detoxified AD exhibited preference toward short-term uncertain reward over safer long-term reward (Noël et al., 2007; Kornreich et al., 2013; Brevers et al., 2014). Hence, one could infer that the occurrence of such impaired decisionmaking processes might hamper ADs' ability to exert willpower, that is, to decide based on both short-term and long-term consequences of an action (Bechara, 2005; Noël et al., 2010). 
One limitation of these studies is that they focus on situations where decision outcomes only affect the deciding agent. Hence, those findings cannot be generalized to situations that involve social interactions, that is, social decision-making that affects others as well as the individual who makes the choice (Fehr and Camerer, 2007; Rilling and Sanfey, 2011). Abnormal social decision-making has been evidenced in alcohol dependence through the use of "moral dilemmas," which trigger a conflict between what is good for the majority (utilitarian judgment) and emotional factors (Greene et al., 2001). Compared to healthy controls, $\mathrm{AD}$ would be more prone to choose the rational options (i.e., utilitarian moral judgments) when faced with emotionally salient moral personal dilemmas (Carmona-Perera et al., 2012, 2013, 2014; Khemiri et al., 2012; but see Kornreich et al., 2013). Moreover, by contrast to control participants, AD showed a blunted emotional reactivity (estimated with heart rate response) to moral personal dilemmas (Carmona-Perera et al., 2013). These findings suggest that $\mathrm{AD}$ failed to engage emotional aversive reactions within situations of social interactions that involve personal moral violations for others.

Impaired social-decision making has also recently been evidenced in $\mathrm{AD}$ through a measure of sensitivity to unfairness. Indeed, a recent study (Brevers et al., 2013) has shown that, as compared to healthy controls, ADs were more prone to reject unfair offers during the ultimatum game (UG; Güth et al., 1982; Sanfey et al., 2003). The context is very different from the studies involving moral dilemmas, as in the UG paradigm, the subjects are confronted with unfair situations directed toward themselves, rather than involving other persons described in theoretical stories. In the UG, two players (a proposer and a responder) have to divide a sum of money in a single trial. If the responder accepts the offer made by the proposer, the deal is validated. On the other hand, if the responder rejects the offer, neither player gets anything. According to rational economic agent, the only way for the responder to maximize profits during the $U G$ is to always accept the offer (Haselhuhn and Mellers, 2005; Hewig et al., 2011). Nevertheless, researchers have constantly observed that individuals are unwilling to accept inequitable UG offers, as compared to fair or medium-fair offers (Camerer and Thaler, 1995; Haselhuhn and Mellers, 2005; Knafo et al., 2008).

Findings from recent studies suggest that the rejection of unfair UG offers is modulated by (negative) emotional processes. More specifically, possible explanation for these seemingly "irrational" rejection is that negative emotions, such as unfairness, might drive participants to punish the proposer rather than making an utilitarian choice (Pillutla and Murnighan, 1996; Fehr and Gächter, 2002). This assumption is in line with the theory of altruistic punishment (Trivers, 1971; Fehr and Gächter, 2000, 2002), which advances that people are willing to punish noncooperators in order to enhance reciprocity in cooperation transactions, even at personal costs to the punisher (as in the case of rejecting an UG offer). Consistent with this theoretical view, inequitable proposals were found to elicit a stronger neural activation in emotion-related brain regions (Sanfey et al., 2003; Koenigs and Tranel, 2007; Feng et al., 2014) and a greatermagnitude of skin conductance response (SCR; van't Wout et al., 2006; Hewig et al., 2011; but see Osumi and Ohira, 2009).
Moreover, the proportion of rejected unfair UG offers is positively associated with self-reported anger (Pillutla and Murnighan, 1996) and with greater SCR (Civai et al., 2010; Wu et al., 2013).

The present study aimed to examine the association between emotional state and unfairness sensitivity in $\mathrm{AD}$ and healthy controls while performing the UG. Emotional state was indexed by skin conductance reactivity amplitude. Electrodermal response is a valid measure of emotional activation (Bouscein, 1992; Canli and Lesch, 2007), which has also been found to be sensitive to the degree of unfairness associated with UG offers (van't Wout et al., 2006; Civai et al., 2010; Hewig et al., 2011; Wu et al., 2013). We hypothesized that (i) AD reject unfair offers more frequently than controls; (ii) in both $\mathrm{AD}$ and controls, facing unfair offers will trigger higher SCR, as compared with medium-fair and fair offers; (iii) AD exhibit higher SCR than controls when facing unfair offers.

\section{Materials and Methods}

\section{Participants and Recruitment}

All subjects were adults. Demographics for the two groups are presented in Table 1. All participants provided informed consent. The study was fully approved by the Ethics Committee of the Brugmann University Hospital and conducted in accordance with the Declaration of Helsinki. For the AD group, medical histories were obtained by interview by a board-certified psychiatrist.

Twenty-six AD participants were recruited for this study from the Alcohol Detoxification Unit of the Brugmann University Hospital (Brussels). They were tested in their third week of alcohol detoxification (i.e., the week before their detoxification program end). They all received complete medical, neurological and psychiatric examinations at the time of the selection. $\mathrm{AD}$ participants were all diagnosed (made by $\mathrm{PV}$ and $\mathrm{CH}$, board-certified CHU-Brugmann psychiatrists) with alcohol dependence according to DSM-V (American Psychiatric

TABLE 1 | Demographic data means and standard deviations for the alcohol-dependents (ADs) and controls.

\begin{tabular}{lll}
\hline & AD & Control \\
\hline$N$ & 26 & 32 \\
Age (years) & $42.53(8.83)$ & $41.25(10.08)$ \\
Gender (male/female) & $19 / 7$ & $24 / 8$ \\
Education (years) & $13.42(3.17)$ & $17.06(3.14)^{\star \star \star \star}$ \\
Duration of alcohol abuse (years) & $13.50(10.99)$ & - \\
Mean alcohol use (drinks/day) & $15.03(10.81)$ & $1.96(1.82)^{\star \star \star \star}$ \\
Number of prior hospitalizations & $2.61(2.51)$ & - \\
for alcohol detoxification & & \\
Tobacco use (number of cigarettes per day) & $24.15(16.81)$ & $3.43(5.76)^{\star \star \star \star}$ \\
AUDIT & $28.89(5.20)$ & $2.59(3.87)^{\star \star \star \star}$ \\
BDI & $10.96(6.23)$ & $3.56(3.44)^{\star \star \star \star}$ \\
STAI-S & $54.27(12.25)$ & $32.46(14.02)^{\star \star \star \star}$ \\
STAI-T & $42.61(14.02)$ & $30.03(9.65)^{\star \star \star \star}$
\end{tabular}

Values shown are the mean and standard deviation on each measure. AUDIT, Alcohol Use Disorders Identification Test; BDI, Beck Depression Inventory; STAI-S, State subscale of the State-Trait Anxiety Inventory; STAI-T, Trait subscale of the State-Trait Anxiety Inventory. ${ }^{* * * *}$ t-test $p<0.0001$. 


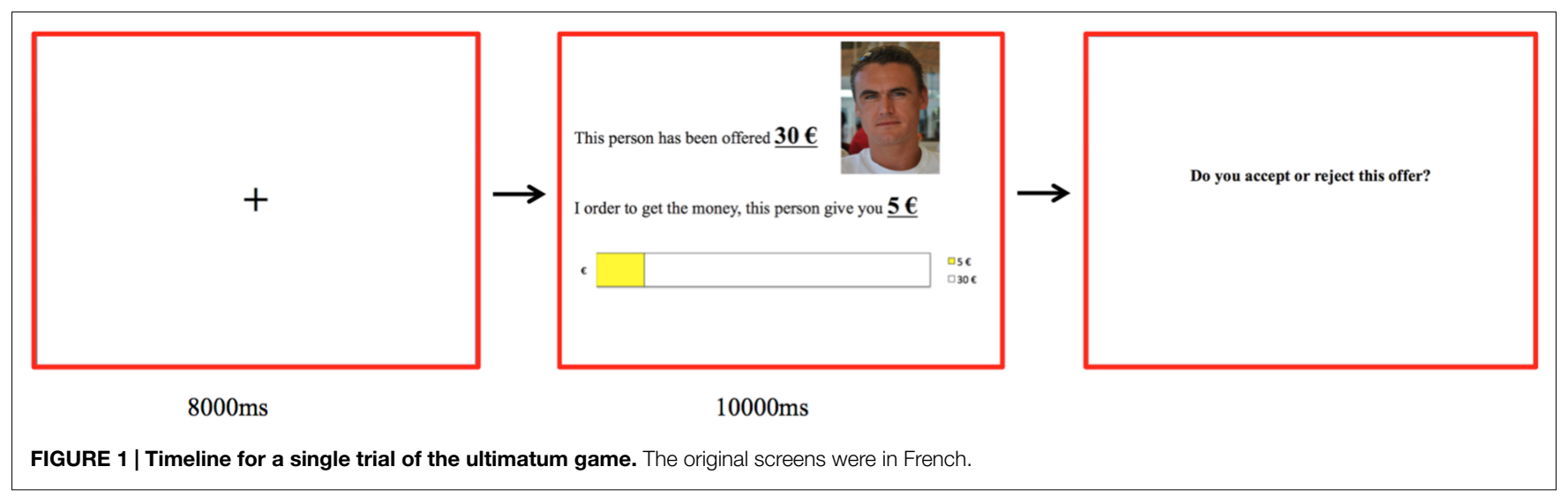

Association, 2013) criteria and confirmed by the Alcohol Use Disorders Identification Test (AUDIT; Saunders et al., 1993). We excluded any subject who reported a lack of comprehension of French language, or who had evidence of schizophrenia and other psychotic disorders, bipolar disorders, polysubstancerelated disorders, pathological gambling and overt cognitive dysfunction (based on DSM-V interviews, clinical observations, and anamnestic information collected by PV and $\mathrm{CH}$ ). Reasons for exclusion were other current DSM-IV Axis I diagnoses, a history of significant medical illness, head injury resulting in a loss of consciousness for longer than $30 \mathrm{~min}$ that might have affected the central nervous system, use of other psychotropic drugs or substances that influence cognition and overt cognitive dysfunction. Subjects were examined after they had abstained from alcohol for a minimum of 18 days and at least 5 days after a standard detoxification period. The detoxification regimen consisted of $\mathrm{B}$ vitamins and decreasing doses of sedative medication (diazepam). All received complete medical, neurological, and psychiatric evaluations prior to enrolment in the study.

Thirty-two control participants, similar for sex and age, were recruited by word of mouth from healthy community members; they were not paid for their participation. Exclusion criteria were a present Axis I psychiatric diagnosis; substance-use disorder during the year before enrollment in the study; or consumption of more than four standard alcoholic drinks per day for longer than 1 month.

For each participant, current clinical status of depression and anxiety levels were rated with the Beck Depression Inventory (Beck et al., 1961) and the Spielberger State-Trait Anxiety Inventory (STAI-S, STAI-T; Spielberger, 1983). Participants completed the STAI-S just prior performing the UG task.

\section{Task}

Participants played as responders in a series of 54 single round trials of the UG via computer interface. Before the game started, participants were given detailed verbal explanations and confirmed verbally that they understood the game. No real monetary amount was awarded to the participants. At the beginning of each trial, a white-colored, cross-shaped fixation point was presented for $8000 \mathrm{~ms}$ in order to allow autonomic activity from the previous trial to recover to baseline. Next, they saw the amount of the stake and the amount proposed by the partner for $10000 \mathrm{~ms}$ (see Figure 1). During this time, a picture of the proposer's face was displayed in the upper right of the computer screen (see Figure 1). Then, a "response cue" was displayed and participants indicated whether they accepted or reject the offer by pressing one of two buttons (labeled "accept" or "reject") on a button box.

Photographs of 54 faces (27 male, 27 female, Caucasian, with a neutral expression; taken from Brevers et al., 2013) were randomly matched with the offers. There were 18 fair offers (proposition of 40,45 , or $50 \%$ of the stake to the responder), 18 medium-fair offers (proposition of 27,30 , or $33 \%$ of the stake to the responder) and 18 unfair offers (proposition of 17,20 , or $22 \%$ of the stake to the responder). During each session, the order of the offers was randomized.

\section{Apparatus}

The UG paradigm was programmed using E-prime 2.0 Professional (Psychology Software Tools, Sharpsburg, PA, USA). Electrodermal activity (EDA) was recorded during the task using a BIOPAC MP150 (Biopac Systems, Santa Barbara, CA, USA). The BIOPAC was connected to a stimulus delivery computer and a second administrator computer running AcqKnowledge v3.9.0. Events occurring on the stimulus delivery computer were synchronized to the psychophysiology recording using digital channels. EDA was measured using two grounded $\mathrm{Ag}-\mathrm{AgCl}$ electrodes (a BIOPAC TSD203 transducer with a GSR100C amplifier module; gain $=5 \mathrm{~V}$, low pass filter $1.0 \mathrm{~Hz}$, high pass filters DC), secured on the distal phalange of the index and middle fingers of the non-dominant hand. Isotonic paste (BIOPAC Gel 101, with recommended $\mathrm{NaCl}$ concentration of $0.05 \mathrm{M}$ ) was used as the electrolyte. The EDA signal was transformed into micro-Siemens units $(\mathrm{mS})$ in Acqknowledge.

\section{Data Preprocessing}

Physiological data were analyzed offline in AcqKnowledge (Biopac Systems, Santa Barbara, CA, USA). SCR amplitude was defined as the change from the baseline to the peak of the response started within a $0.5-4 \mathrm{~s}$ duration following offer onset. Behavioral (mean proportion of acceptance) and physiological (mean SCR) was not normally distributed, at each level of type of offers 
TABLE 2 | Descriptive statistics of the proportion of offers accepted for alcohol-dependent and control participants with unfair, medium fair, and fair offers.

\begin{tabular}{lllll}
\hline & & \multicolumn{3}{c}{ Percentiles } \\
\cline { 3 - 4 } & & 25th & Median & 75th \\
\hline \multirow{2}{*}{ Controls } & Fair & 0.77 & 100 & 100 \\
& Medium-fair & 0.12 & 0.79 & 100 \\
Alcohol-dependent & Unfair & 0.08 & 0.50 & 100 \\
& Fair & 0.48 & 0.83 & 100 \\
& Medium-fair & 0.17 & 0.33 & 0.58 \\
& Unfair & 0.00 & 0.17 & 0.50 \\
& & & & \\
\end{tabular}

(fair, medium-fair, unfair) and for each decision type (accept vs. reject). Attempts of $\log$ transformation $(\log [S C R+1])$ had no significant effect on the normality of behavioral and physiological data. Therefore, non-parametric statistical tests were performed to analyze behavioral decision and SCR amplitude during the UG.

\section{Results}

\section{Current Clinical Status}

Independent samples $t$-tests revealed that $\mathrm{AD}$ participants had lower scores of education, higher scores of depression, trait anxiety and state anxiety (prior to testing). These results are shown in Table 1. However, we found no correlation (Spearman rank) between UG (behavioral and electrodermal) data and the scores of education, depression, trait and state anxiety, for the whole sample $(N=58)$, within the control $(n=32)$ or the alcohol dependence group $(n=26)$. We observed no significant effect of gender (for the whole sample and within the AD and control groups separately) on UG (behavioral and electrodermal) data. There was also no relationship between UG data in the AD group and duration of consumption or mean consumption per day.

\section{Behavioral Performances}

A Friedman test was performed separately in each group in order to examine the effect of the type of offers (fair vs. mediumfair vs. unfair) on the proportion of acceptance (see Table 2, for descriptive statistics). These analyses showed that there were significant differences, in each group, between the fair and the medium-fair offers $(p<0.0001)$, between the fair and the unfair offers $(p<0.0001)$. In the control group, we found no significant difference between the medium-fair and the unfair offers, $\chi^{2}(1,32)=0.69, p=0.40$. By contrast, in the alcohol dependence group, there was a significant difference between the medium-fair and the unfair offers $\left[\chi^{2}(1,26)=4.00, p=0.046\right]$, indicating that $\mathrm{AD}$ participants reject more unfair offers than medium fair offers.

Mann-Whitney $U$ tests were then performed to examine between-groups differences on the proportion of acceptance according to the type of offers. There was a significant difference between AD (Mean Rank = 24.35) and controls (Mean Rank $=33.69$ ) for the unfair offers (Mann-Whitney $U$ statistic $=282.00, Z=-2.132, p=0.033$ ), indicating that $\mathrm{AD}$ decided to reject unfair offers more frequently than controls (see Table 2, for descriptive statistics). There was no significant difference between $\mathrm{AD}$ and control groups for the medium-fair (Mann-Whitney U statistic $=306.50, Z=-1.741, p=0.082$ ) and the fair offers (Mann-Whitney U statistic $=323.00, Z=-1.546$, $p=0.122$ ). In addition, in order to estimate the interaction between group and type of offers, Mann-Whitney $U$ tests were performed with scores computing the difference of acceptance rate between each type of offers (i.e., unfair minus fair; unfair minus medium-fair; medium-fair minus fair). These analyses did not reach any significant results, all $p>0.05$.

\section{Skin Conductance Response}

A Friedman test was performed separately in each group in order to examine the effect of the type of offers (fair vs. medium-fair vs. unfair) on SCR (in $\mathrm{mS}$ ). These analyses showed no significant SCR differences, in each group (all $p>0.05$ ), between the fair (control group: mean $=0.315$, median $=0.254,25$ th $=0.09$, 75 th $=0.42$; alcohol group: mean $=0.41$, median $=0.33$, 25 th $=0.138,75$ th $=0.583$ ), the medium-fair (control group: mean $=0.296$, median $=0.18,25$ th $=0.10,75$ th $=0.47$; alcohol group: mean $=0.288$, median $=0.19,25$ th $=0.008$, 75 th $=0.413$ ) and the unfair offers (control group: mean $=0.292$, median $=0.33,25$ th $=0.071,75$ th $=0.45$; alcohol group: mean $=0.41$, median $=0.33,25$ th $=0.138,75$ th $=0.583)$. We then performed correlation analyses (Spearman Rho) between SCR and proportion of acceptance, for each types of offer and each group separately. These analyses showed that, in the alcohol dependence group, proportion of acceptance is negatively correlated with SCR for the unfair offers (Spearman Rho $=-0.41$, $p=0.04$; see Figure 2). This association did not reach significance in the control group. We observed no significant correlation for the fair and medium fair offers, in both control and AD groups.

Mann-Whitney $U$ tests were then performed to examine between-groups differences on SCR according to the type of offers (fair, medium-fair, or unfair). These analyses showed no significant difference between $\mathrm{AD}$ and controls (all $p>0.05)$. Additional exploratory Mann-Whitney $\mathrm{U}$ tests were performed to examine between-groups differences on SCR according to decision types (accept or reject) for each type of offers (fair, medium-fair, unfair). These analyses revealed a significant difference between $\mathrm{AD}$ participants $(n=23$; average rank $=21.75)$ and controls $(n=20$; average rank $=14.44)$ for the rejection of the unfair offer (Mann-Whitney $U$ statistic $=95.00$, $Z=-2.07, p=0.038$ ), indicating that $\mathrm{AD}$ (mean $=0.488$, median $=0.445,25$ th $=0.207,75$ th $=0.589$ ) exhibited a higher SCR amplitude before rejecting unfair offers, as compared to controls (mean $=0.28$, median $=0.345,25$ th $=0.075$, 75 th $=0.465)$. No other significant difference was observed.

\section{Discussion}

In the present study, we examined the association between emotional state and unfairness sensitivity in recently abstinent $\mathrm{AD}$ individuals and in healthy controls while performing an UG. Emotional state was measured by autonomic reactivity as reflected by SCR.

At a behavioral level, $\mathrm{AD}$ rejected unfair offers more frequently than controls, which replicate findings from the previous 


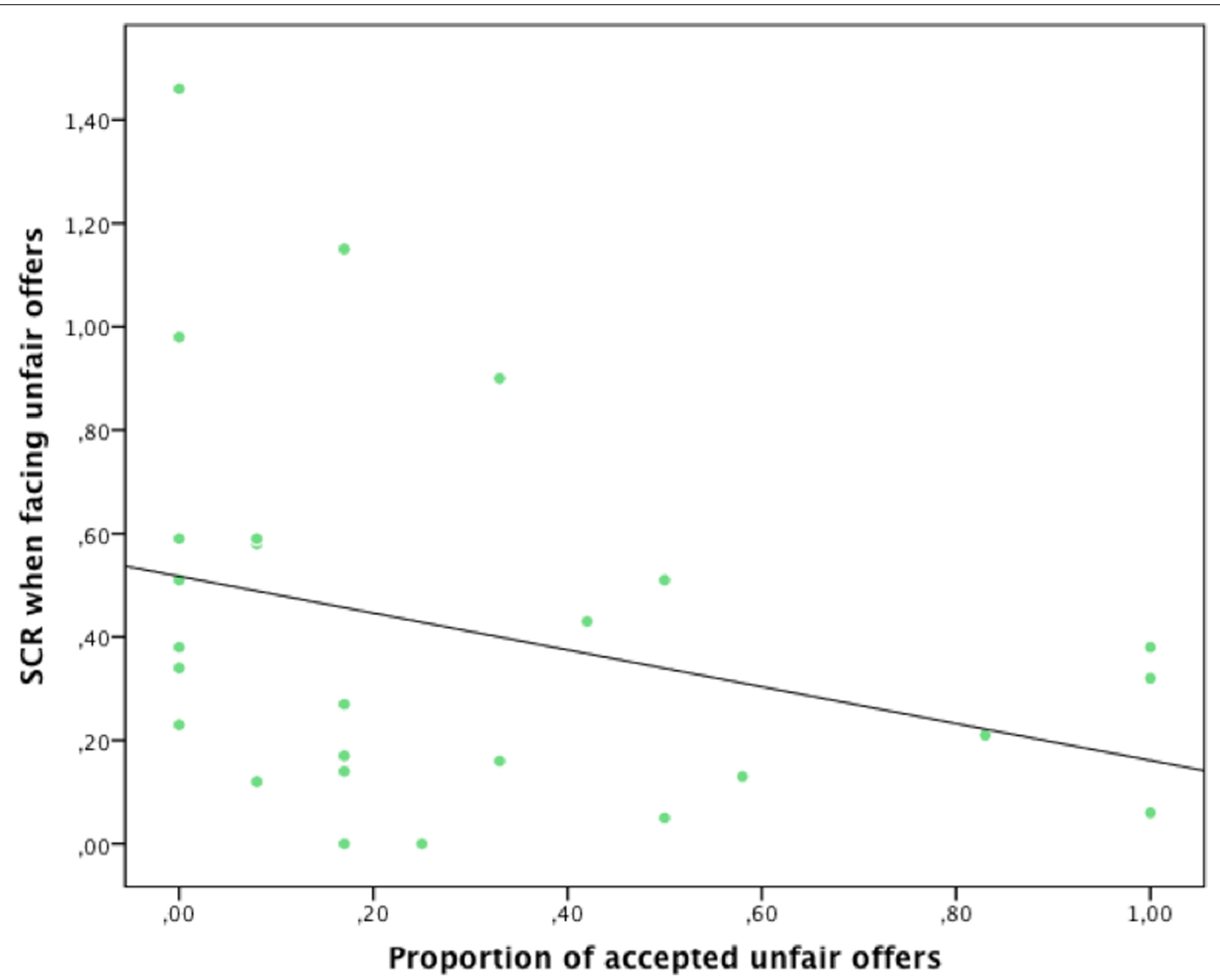

FIGURE 2 | In the alcohol-dependent group $(n=26)$, proportion of accepted unfair offers was negatively correlated with SCR amplitude triggered by unfair offers (Spearman Rho $=-0.41, p=0.04$ ).

UG study in alcohol-dependence (Brevers et al., 2013). At a psychophysiological level, the proportion of accepted unfair UG offers was negatively correlated with SCR, but in the AD group only. Hence, deciding to accept or reject an unfair offer during the UG was influenced by emotional psychophysiological reactivity in $\mathrm{AD}$, but not in controls. In addition, AD exhibited higher SCR than controls for unfair offers, but only prior to rejecting the offer. Importantly, despite its statistical significance, the negative correlation between ADs' proportion of accepted unfair UG offers and SCR remains moderate. In addition, between-groups analyses did not include participants who accepted all unfair offers (12 controls and three AD participants) and the number of observations (e.g., rejected vs. accepted offers) differed for each participants. Hence, current findings are preliminary and have to be interpreted with caution.

Present findings suggest that, in AD: the higher the SCR, the higher the rejection of unfair offers. Hence, because skin conductance reflects sympathetic tone (Bouscein, 1992; van't Wout et al., 2006), the association between SCR changes and UG rejection rates might reflect increased affective-arousal activity when facing unfair UG offers in $\mathrm{AD}$. In other words, present results suggest that unfair offers triggered an emotional state in $\mathrm{ADs}$ and, that as a consequence, they "punished" proposers by rejecting the offer, thus depriving the proposer of their greater share of the money (Nowak et al., 2000). By contrast, nonalcoholics were better able to act as rational economic agents (at both behavioral and psychophysiological levels) during the UG, which results in maximizing their monetary profits. However, in the present study, we used only one marker of emotional state (i.e., SCR). Hence, we cannot exclude that UG offers did not trigger significant emotional state in controls. In addition, present data offers no information on the valence associated with the SCR. Thus, we cannot infer that unfair offers triggered negative emotional state in $\mathrm{AD}$. In this context, one option for future studies would be to make participants complete (after all trials of the UG) a subjective ratings of the emotional valence (e.g., from $1 /$ negative to $9 /$ positive) that they had experienced in each condition (i.e., fair, medium-fair, unfair) while playing the role of responder (e.g., Hewig et al., 2011).

Importantly, contradictory to our hypotheses and inconsistently with previous studies (van't Wout et al., 2006; Hewig et al., 2011; but see Osumi and Ohira, 2009), SCR was not modulated by the degree of unfairness associated with UG offers. Moreover, the absence of a significant correlation between SCR and the proportion of accepted offers in control differs from previous studies which showed that the rejection of unfair UG offers is associated with greater SCR (Civai et al., 2010; Wu et al., 2013). One explanation for these discrepancies is that 
participants recruited for the present study where older (i.e., adults with age ranging from 20 to 51 years) than participants recruited in previous UG psychophysiological studies (i.e., young adults with mean age ranging from 20.10 to 23.56 years; van't Wout et al., 2006; Osumi and Ohira, 2009; Civai et al., 2010; Hewig et al., 2011; Wu et al., 2013). Indeed, multiple lines of research have demonstrated that age is associated with lower negative affectivity (Pillutla and Murnighan, 1996). For instance, laboratory studies demonstrated that age is associated with lower negative experiential and physiological reactivity to laboratory emotion inductions (Levenson, 2000; Tsai et al., 2000; LabouvieVief et al., 2003). Hence, as compared to young adults, facing unfair offers might have less emotional impact on middle-aged adults, which could increase their level acceptance toward unequal offers. Another possible explanation for these findings is that, in the present study, no real money was awarded to the participants. As a result, the use of hypothetical money could have lowered participants' emotional engagement during the UG task and decreased the validity of the observed SCR. Hence, one direction for future studies would be to examine the emotional correlates of unfairness sensitivity in alcohol dependence through the use of an experiential UG played with real money.

An implication of present findings is that $\mathrm{AD}$ might have difficult to cope with unfair situations triggered by (e.g.) social interactions. Nevertheless, additional studies are needed in order to examine whether-emotional and behavioral-reactivity to unfairness during the UG could predict relapse in abstinent AD. Indeed, sensitivity to frustration has been reported to impact alcohol consumption and relapse in abstinent $\mathrm{AD}$ patients (Muraven et al., 2002; Baars et al., 2013; Winward et al., 2014). Future studies should also examine psychophysiological responses to UG offers in different subtypes of AD. For instance, patients with high interoceptive awareness are at elevated risk of negative mood or stress-induced relapse (Wiens, 2005; Verdejo-Garcia et al., 2012). As a result, these individuals might exhibit increased emotional and behavioral reactivity to unfairness during the UG. Indeed, it has been evidenced that SCR responses is associated with UG offers' rejection in people with high interoceptive abilities but not in individuals with poor

\section{References}

American Psychiatric Association. (2013). Diagnostic and Statistical Manual of Mental Disorders, 5th Edn. Arlington, VA: American Psychiatric Publishing.

Baars, M. Y., Müller, M. J., Gallhofer, B., and Netter, P. (2013). Relapse (number of detoxifications) in abstinent male alcohol-dependent patients as related to personality traits and types of tolerance to frustration. Neuropsychobiology 67, 241-248. doi: 10.1159/000350483

Bechara, A. (2005). Decision making, impulse control and loss of willpower to resist drugs: a neurocognitive perspective. Nat. Neurosci. 8, 1458-1463. doi: $10.1038 / \mathrm{nn} 1584$

Beck, A. T., Ward, C. H., Mendelson, M., Mock, J., and Erbaugh, J. (1961). An inventory for measuring depression. Arch. Gen. Psychiatry 4, 561-571. doi: 10.1001/archpsyc.1961.01710120031004

Bouscein, W. (1992). Electrodermal Activity. New York, NY: Plenum.

Brevers, D., Bechara, A., Cleeremans, A., Kornreich, C., Verbanck, P., and Noël, X. (2014). Impaired decision-making under risk in individuals with alcohol dependence. Alcohol. Clin. Exp. Res. 38, 1924-1931. doi: 10.1111/acer.12447

Brevers, D., Noël, X., Ermer, E., Dabiri, D., Verbanck, P., and Kornreich, C. (2013). Unfairness sensitivity and social decision-making in individuals with alcohol interoceptive sensitivity (Dunn et al., 2012; see also Kirk et al., 2011). It would be also interesting to examine whether Cloninger Type 1-anxiety prone-alcoholism differ from Cloninger Type 2-impulsive-alcoholism at the UG. For instance, it has been highlighted that people differing in levels of anxiety showed distinct behavior patterns and autonomic neural responses during the UG (i.e., SCR to inequitable offers was correlated with levels of anxiety in individuals with high-trait anxiety, but not in the individuals with low-trait anxiety), and that impulsivity might be an additional moderating factors in UG offers acceptance (Wu et al., 2013). Brain-imaging studies could also shed light on specific processes underlying impaired social decision-making in alcohol-dependence. For instance, insular activation plays a key role in representing somatic states used to simulate the potential negative consequences of an action (Damasio, 1994; Craig, 2002, 2009), such as when people reject unfair UG offers at substantial cost to themselves (Sanfey et al., 2003; Grecucci et al., 2012; Harle et al., 2012). Hence, it would be interesting to examine if increased unfairness sensitivity in $\mathrm{AD}$ is underlined by increased insular activation during the UG.

In sum, $\mathrm{AD}$ exhibited a lower rate of acceptation for unfair offers during an UG task. Moreover, ADs' tendency to accept or reject unfair UG offers was associated with the level of electrodermal response triggered by the offer. This association was not found in control participants. These findings offer a first evidence of hampered emotion regulation in alcohol dependence when facing a social context of unfairness. Nevertheless, these results remain preliminary and additional studies are needed in order to further examine the impact of emotion on unfairness sensitivity in $\mathrm{AD}$.

\section{Acknowledgments}

DB is a Post-Doctoral Research Scholar supported by the National Center for Responsible Gaming (NCRG; Early Stage Investigator Grant). $\mathrm{XN}$ is Research Associate at the Belgium Fund for Scientific Research (F.R.S./FNRS). The authors thank Sabrina Rodriguez Lopez and Charlotte Hinnekens for their help in recruiting participants.

dependence: a preliminary study. Drug Alcohol Depend. 133, 772-775. doi: 10.1016/j.drugalcdep.2013.08.013

Camchong, J., Endres, M., and Fein, G. (2014). Decision making, risky behavior, and alcoholism. Handb. Clin. Neurol. 125, 227-236. doi: 10.1016/B978-0-44462619-6.00014-8

Camerer, C. F., and Thaler, R. (1995). Anomalies: dictators, ultimatums, and manners. J. Econ. Perspect. 9, 209-219. doi: 10.1257/jep.9.2.209

Canli, T., and Lesch, K. P. (2007). Long story short: the serotonin transporter in emotion regulation and social cognition. Nat. Neurosci 10, 1103-1109. doi: $10.1038 / \mathrm{nn} 1964$

Carmona-Perera, M., Clark, L., Young, L., Perez-Garcia, M., and Verdejo-Garcia, A. (2014). Impaired decoding of fear and disgust predicts utilitarian moral judgment in alcohol-dependent individuals. Alcohol. Clin. Exp. Res. 38, 179-185. doi: 10.1111/acer.12245

Carmona-Perera, M., Reyes del Paso, G. A., Pérez-García, M., and VerdejoGarcía, A. (2013). Heart rate correlates of utilitarian moral decision-making in alcoholism. Drug Alcohol Depend. 133, 413-419. doi: 10.1016/j.drugalcdep. 2013.06.023

Carmona-Perera, M., Verdejo-García, A., Young, L., Molina-Fernández, A., and Pérez-García, M. (2012). Moral decision-making in polysubstance 
dependent individuals. Drug Alcohol Depend. 126, 389-392. doi: 10.1016/j.drugalcdep.2012.05.038

Civai, C., Corradi-Dell'Acqua, C., Gamer, M., and Rumiati, R. I. (2010). Are irrational reactions to unfairness truly emotionally-driven? Dissociated behavioural and emotional responses in the ultimatum game task. Cognition 114, 89-95. doi: 10.1016/j.cognition.2009.09.001

Craig, A. D. (2002). How do you feel? Interoception: the sense of the physiological condition of the body. Nat. Rev. Neurosci. 3, 655-666. doi: 10.1038/nrn894

Craig, A. D. (2009). How do you feel-now? The anterior insula and human awareness. Nat. Rev. Neurosci. 10, 59-70. doi: 10.1038/nrn2555

Damasio, A. R. (1994). Descartes' Error: Emotions, Reason, and the Human Brain. New York, NY: Avon Books.

Dunn, B. D., Evans, D., Makarova, D., White, J., and Clark, L. (2012). Gut feelings and the reaction to perceived inequity: the interplay between bodily responses, regulation and perception shapes the rejection of unfair offers on the ultimatum game. Cogn. Affect. Behav. Neurosci. 12, 419-429. doi: 10.3758/s13415-0120092-z

Fehr, E., and Camerer, C. F. (2007). Social neuroeconomics: the neural circuitry of social preferences. Trends Cogn. Sci. 11, 419-427. doi: 10.1016/j.tics.2007.09.002

Fehr, E., and Gächter, S. (2000). Cooperation and punishment in public goods experiments. Am. Econ. Rev. 90, 980-994. doi: 10.1257/aer.90.4.980

Fehr, E., and Gächter, S. (2002). Altruistic punishment in humans. Nature 415, 137-140. doi: 10.1038/415137a

Feng, C., Luo, Y. J., and Krueger, F. (2014). Neural signatures of fairness-related normative decision making in the ultimate game: a coordinate-based metaanalysis. Hum. Brain Mapp. 36, 591-602. doi: 10.1002/hbm.22649

Grecucci, A., Giorgetta, C., Van't Wout, M., Bonini, N., and Sanfey, A. G. (2012). Reappraising the ultimatum: an fMRI study of emotion regulation and decision making. Cereb. Cortex 23, 399-410. doi: 10.1093/cercor/bhs028

Greene, J. D., Sommerville, R. B., Nystrom, L. E., Darley, J. M., and Cohen, J. D. (2001). An fMRI investigation of emotional engagement in moral judgment. Science 293, 2105-2108.

Güth, W., Schmittenberger, R., and Schwarze, B. (1982). An experimental analysis of ultimatum bargaining. J. Econ. Behav. Organ. 3, 367-388. doi: 10.1016/01672681(82)90011-7

Harle, K. M., Chang, L. J., van 't Wout, M., and Sanfey, A. G. (2012). The neural mechanisms of affect infusion in social economic decisionmaking: a mediating role of the anterior insula. Neuroimage 61, 32-40. doi: 10.1016/j.neuroimage.2012.02.027

Haselhuhn, M. P., and Mellers, B. A. (2005). Emotions and cooperation in economic games. Brain Res. Cogn. Brain Res. 23, 24-33. doi: 10.1016/j.cogbrainres.2005.01.005

Hewig, J., Kretschmer, N., Trippe, R. H., Hecht, H., Coles, M. G. H., Holroyd, C. B., et al. (2011). Why humans deviate from rational choice. Psychophysiology 48, 507-514. doi: 10.1111/j.1469-8986.2010.01081.x

Khemiri, L., Guterstam, J., Franck, J., and Jayaram-Lindsrtröm, N. (2012). Alcohol dependence associated with increased utilitarian moral judgment: a case control study. PLoS ONE 7:e39882. doi: 10.1371/journal.pone.0039882

Kirk, U., Downar, J., and Montague, R. P. (2011). Interoception drives increased rational decision-making in meditators playing the ultimatum game. Front. Neurosci. 5:49. doi: 10.3389/fnins.2011.00049

Knafo, A., Israel, S., Darvasi, A., Bachner-Melman, R., Uzefovsky, F., Cohen, L., et al. (2008). Individual differences in allocation of funds in the dictator game associated with length of the arginine vasopressin 1a receptor RS3 promoter region and correlation between RS3 length and hippocampal mRNA. Genes Brain Behav. 7, 266-275. doi: 10.1111/j.1601-183X.2007. 00341.x

Koenigs, M., and Tranel, D. (2007). Irrational economic decision-making after ventromedial prefrontal damage: evidence from the ultimatum game. $J$. Neurosci. 27, 951-956. doi: 10.1523/JNEUROSCI.4606-06.2007

Kornreich, C., Brevers, D., Ermer, E., Hanak, C., Verbanck, P., Campanella, S., et al. (2013). Polysubstance dependent patients display a more utilitarian profile in moral decision-making than alcohol-dependent patients, depressive patients and controls. Drug Alcohol Depend. 132, 434-440. doi: 10.1016/j.drugalcdep.2013.03.005

Labouvie-Vief, G., Lumley, M. A., Jain, E., and Heinze, H. (2003). Age and gender differences in cardiac reactivity and subjective emotion responses to emotional autobiographical memories. Emotion 3, 115-126. doi: 10.1037/15283542.3.2.115
Levenson, R. W. (2000). "Expressive, physiological, and subjective changes in emotion across adulthood," in Psychology and the Aging Revolution: How We Adapt to Longer Life, eds S. H. Qualls and N. Abeles (Washington, DC: American Psychological Association), 123-140.

Muraven, M. l., Collins, R. L., and Nienhaus, K. (2002). Self-control and alcohol restraint: an initial application of the self-control strength model. Psychol. Addict. Behav. 16, 113-120. doi: 10.1037/0893-164X.16.2.113

Noël, X., Bechara, A., Brevers, D., Verbanck, P., and Campanella, S. (2010). Alcoholism and the loss of willpower: a neurocognitive perspective. $J$. Psychophysiol. 24, 240-248. doi: 10.1027/0269-8803/a000037

Noël, X., Van der Linden, M., d'Acremont, M., Bechara, A., Dan, B., Hanak, C., et al. (2007). Alcohol cues increase cognitive impulsivity in individuals with alcoholism. Psychopharmacology 192, 291-298. doi: 10.1007/s00213-0060695-6

Nowak, M. A., Page, K. M., and Sigmund, K. (2000). Fairness versus reason in the ultimatum game. Science 289, 1773-1775. doi: 10.1126/science.289.5485. 1773

Osumi, T., and Ohira, H. (2009). Cardiac responses predict decisions: an investigation of the relation between orienting response and decisions in the ultimatum game. Int. J. Psychophysiol. 74, 74-79. doi: 10.1016/j.ijpsycho.2009.07.007

Pillutla, M. M., and Murnighan, J. K. (1996). Unfairness, anger, and spite: emotional rejections of Ultimatum Offers. Organ. Behav. Hum. Decis. Process. 68, 208-224. doi: 10.1006/obhd.1996.0100

Rilling, J. K., and Sanfey, A. G. (2011). The neuroscience of social decisionmaking. Annu. Rev. Psychol. 62, 23-48. doi: 10.1146/annurev.psych.121208. 131647

Sanfey, A. G., Rilling, J. K., Aronson, J. A., Nystrom, L. E., and Cohen, J. D. (2003). The neural basis of economic decision-making in the ultimatum game. Science 300, 1755-1758. doi: 10.1126/science.1082976

Saunders, J. B., Aasland, O. G., Babor, T. F., de la Fuente, J. R., and Grant, M. (1993). Development of the Alcohol Use Disorders Identification Test (AUDIT): WHO collaborative project on early detection of persons with harmful alcohol consumption-II. Addiction 88, 791-804. doi: 10.1111/j.13600443.1993.tb02093.x

Spielberger, C. (1983). Manual for the State-Trait Anxiety Inventory: STAI (Eorm I). Palo Alto, CA: Consulting Psychologists Press.

Trivers, R. L. (1971). The evolution of reciprocal altruism. Q. Rev. Biol. 46, 35-57. doi: $10.1086 / 406755$

Tsai, J. L., Levenson, R. W., and Carstensen, L. L. (2000). Autonomic, subjective, and expressive responses to emotional films in older and younger Chinese Americans and European Americans. Psychol. Aging 15, 684-693. doi: 10.1037/0882-7974.15.4.684

van't Wout, M., Kahn, R. S., Sanfey, A. G., and Aleman, A. (2006). Affective state and decision-making in the ultimatum game. Exp. Brain Res. 169, 564-568. doi: 10.1007/s00221-006-0346-5

Verdejo-Garcia, A., Clark, L., and Dunn, B. D. (2012). The role of interoception in addiction: a critical review. Neurosci. Biobehav. Rev. 36, 1857-1869. doi: 10.1016/j.neubiorev.2012.05.007

Wiens, S. (2005). Interoception in emotional experience. Curr. Opin. Neurobiol. 18, 442-447. doi: 10.1097/01.wco.0000168079.92106.99

Winward, J. L., Bekman, N. M., Hanson, K. L., Lejuez, C. W., and Brown, S. A. (2014). Changes in emotional reactivity and distress tolerance among heavy drinking adolescents during sustained abstinence. Alcohol. Clin. Exp. Res. 38, 1761-1769. doi: 10.1111/acer.12415

Wu, T., Luo, Y., Broster, L. S., Gu, R., and Luo, Y. J. (2013). The impact of anxiety on social decision-making: behavioral and electrodermal findings. Soc. Neurosci. 8, 11-21. doi: 10.1080/17470919.2012.694372

Conflict of Interest Statement: The authors declare that the research was conducted in the absence of any commercial or financial relationships that could be construed as a potential conflict of interest.

Copyright (c) 2015 Brevers, Noël, Hanak, Verbanck and Kornreich. This is an openaccess article distributed under the terms of the Creative Commons Attribution License (CC BY). The use, distribution or reproduction in other forums is permitted, provided the original author(s) or licensor are credited and that the original publication in this journal is cited, in accordance with accepted academic practice. No use, distribution or reproduction is permitted which does not comply with these terms. 